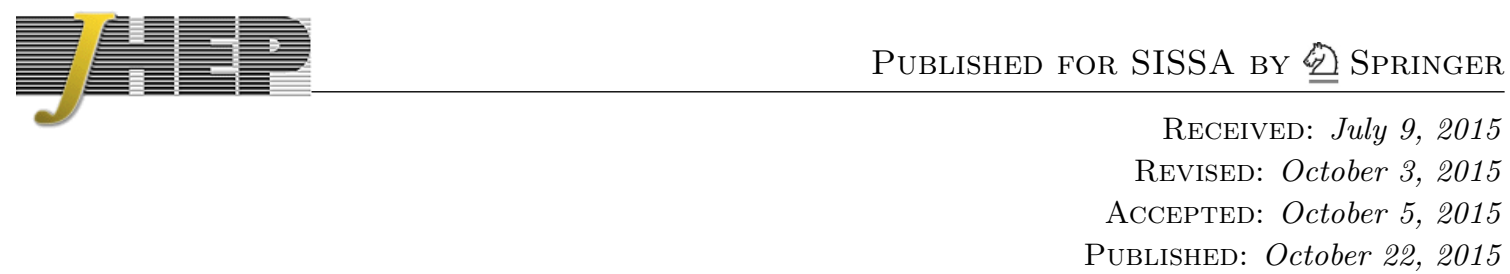

\title{
$\alpha$-attractors: Planck, LHC and dark energy
}

\author{
John Joseph M. Carrasco, ${ }^{a}$ Renata Kallosh $^{b}$ and Andrei Linde ${ }^{b}$ \\ ${ }^{a}$ Institut de Physique Théorique, CEA/DSM/IPhT, CEA-Saclay, \\ 91191 Gif-sur-Yvette, France \\ ${ }^{b}$ SITP and Department of Physics, Stanford University, \\ Stanford, CA 94305 U.S.A. \\ E-mail: dr.jjmc@gmail.com, kallosh@stanford.edu, alinde@stanford.edu
}

ABSTRACT: We develop four-parameter supergravity models of inflation and dark energy, constrained so that $\frac{\delta \rho}{\rho}, n_{s}$ and the cosmological constant $\Lambda$ take their known observable values, but where the mass of gravitino $m_{3 / 2}$ and the tensor-to-scalar ratio $r$ are free parameters. We focus on generalized cosmological $\alpha$-attractor models, with logarithmic Kähler potentials, a nilpotent goldstino and spontaneously broken supersymmetry at the de Sitter minimum. The future data on B-modes will specify the parameter $\alpha$, measuring the geometry of the Kähler manifold. The string landscape idea for dark energy is supported in these models via an incomplete cancellation of the universal positive goldstino and negative gravitino contribution. The scale of SUSY breaking $M$ related to the mass of gravitino in our models is a controllable parameter, independent on the scale of inflation, it will be constrained by LHC data and future collider Energy-frontier experiments.

KEYWORDs: Cosmology of Theories beyond the SM, Supergravity Models

ARXiv EPrint: 1506.01708 


\section{Contents}

1 Introduction 1

2 Review 2

$2.1 \alpha$, and attraction 2

2.2 T and E model attractors, and observables 2

2.3 Stabilizers 3

2.4 Shift symmetry and Z, T, and $\Phi$ variables 4

$3 \quad$ Killing-adapted $\alpha$-attractor supergravity models $\quad 6$

4 Reconstruction models of inflation with SUSY breaking and de Sitter exit

4.1 The simplest T-model with broken SUSY and dS exit 9

$\begin{array}{ll}4.2 & \text { The simplest E-model with broken SUSY and dS exit }\end{array}$

5 General models of inflation with SUSY breaking and dark energy 11

6 Discussion 14

\section{Introduction}

During the next few years we might expect some dramatic new information from B-mode experiments either detecting primordial gravity waves or establishing a new upper bound on $r$, and from LHC discovery/non-discovery of low scale supersymmetry. A theoretical framework to discuss both of these important factors in cosmology and particle physics has been proposed recently. It is based on the construction of new models of chaotic inflation [1, $2]$ in supergravity compatible with the current cosmological data [3, 4] as well as involving a controllable supersymmetry breaking at the minimum of the potential [5-9]. In this paper we will develop supergravity models of inflation motivated by either string theory or extended supergravity considerations, known as cosmological $\alpha$-attractors [10-26]. Here we will enhance them with a controllable supersymmetry breaking and cosmological constant at the minimum. We find this to be a compelling framework for the discussion of the crucial new data on cosmology and particle physics expected during the next few years. Some models of this type were already discussed in [24].

The paper is organized as follows. We begin in section 2 with a brief review of key vocabulary and features of these and related models with references to more in-depth treatments. In section 3 we present the $\alpha$-attractor supergravity models that make manifest an inflaton shift-symmetry by virtue of having the Kähler potential inflaton independent — which we will refer to as Killing-adapted form. Section 4 presents a universal rule: given 
a bosonic inflationary potential of the form $\mathcal{F}^{2}(\varphi)$ one can reconstruct the superpotential $W=\left(S+\frac{1}{b}\right) f(\Phi)$ for the Kähler potentials described in section 3. The resulting models with $f^{\prime}(\varphi)=\mathcal{F}(\varphi)$ have a cosmological constant $\Lambda$ and an arbitrary SUSY breaking $M$ at the minimum. In section 5 we study more general class of models with $W=g(\varphi)+S f((\varphi)$ and the same Kähler potential. For these models it is also possible to get agreement with the Planck data as well as dark energy and SUSY breaking. Moreover, these models have nice properties with regard to initial conditions for inflation, analogous to the ones studied in [27] for models without SUSY breaking and dark energy. We close in section 6 with a summary of what we have accomplished.

\section{Review}

\section{$2.1 \quad \alpha$, and attraction}

There is a key parameter $\alpha$ in these models, for which the Kähler potential $K=-3 \alpha \ln (T+$ $\bar{T})$. It describes the moduli space curvature [11] given by $\mathcal{R}_{K}=-\frac{2}{3 \alpha}$. Another, also geometric, interpretation of this parameter is in terms of the Poincaré disk model of a hyperbolic geometry with the radius $\sqrt{3 \alpha}$, illustrated by the Escher's picture Circle Limit IV $[25,26]$. As clarified in these references, from the fundamental point of view, there are particularly interesting values of $\alpha$ depending on the original theory. From the maximal $\mathcal{N}=4$ superconformal theory, [28-31], one would expect $\alpha=1 / 3$ with $r \approx 10^{-3}$. This corresponds to the unit radius Escher disk [25], as well as a target of the future space mission for B-mode detection, as specified in CORE (Cosmic ORigins Explorer). Some interesting simplifications occur for $\alpha=1 / 9$, which corresponds to the GL model [32-34]. From $\mathcal{N}=1$ superconformal theory [10], one would expect $\alpha=1$ with $r \approx 3 \times 10^{-3}$. Generic $\mathcal{N}=1$ supergravity allows any positive $\alpha$ and, therefore an arbitrary $r$, which has to be smaller than 0.11 to agree with the current data.

\section{$2.2 \quad T$ and $E$ model attractors, and observables}

A simple class of $\alpha$-attractor models, T-models, have a potential $V=\tanh ^{2 n} \frac{\varphi}{\sqrt{6 \alpha}}$ for the canonical inflaton field $\varphi$. These models have the following values of the cosmological observables [10-13] for $\alpha \lesssim O(10)$, where there is an attractor behavior and many models have the same $n$-independent predictions

$$
n_{s}=1-\frac{2}{N}, \quad r=\alpha \frac{12}{N^{2}}, \quad r \approx 3 \alpha \times 10^{-3} .
$$

Once we increase $\alpha$ beyond $O(10)$, expressions for $n_{s}$ and $r$ become somewhat different, see eqs. (5.2)-(5.4) in [12]. In particular, the value of $r$ can be increased significantly, all the way to the predictions of the $\varphi^{2 n}$ models. Even the simplest of these T-models are interesting phenomenologically for cosmology. For these models the parameter $\alpha$ can take any non-zero value; it describes the inverse curvature of the Kähler manifold [11, 13]. The cosmological predictions of these models, for various values of $\alpha$, are shown in figure 1 . As one can see, the line with $n=1$ begins at a point corresponding to the predictions of the simplest quadratic model $\frac{m^{2}}{2} \phi^{2}$ for $\alpha>10^{3}$, and then, for smaller $\alpha$, it rapidly cuts through 


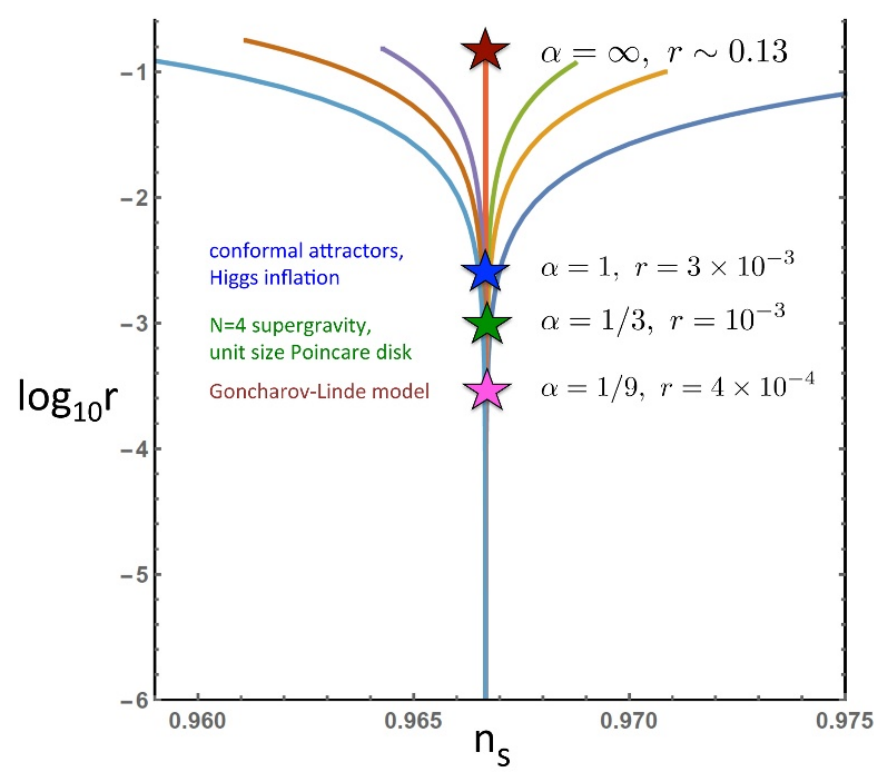

Figure 1. Examples of supergravity T- models with $r$-dependence in logarithmic scale in $r$. For potentials $V=\tanh ^{2 n} \frac{\varphi}{\sqrt{6 \alpha}}$, the predictions of these models interpolate between the predictions of various polynomial models $\varphi^{2 n}$ at very large $\alpha$ and the vertical attractor line for $\alpha \leq O(10)$. When $\alpha \rightarrow \infty$ the models approach the ones with $\varphi^{2 n}$ potentials. This attractor line beginning with the red star corresponds to the predictions of the simplest models $V=\tanh ^{2 n} \frac{\varphi}{\sqrt{6 \alpha}}$ with $n=1$.

the region most favored by the Planck data, towards the predictions of the Higgs inflation model and conformal attractors $r \approx 0.003$ for $\alpha=1$, continues further down towards the prediction $r \approx 0.0003$ of the GL model [32-34] corresponding to $\alpha=1 / 9$, and then the line goes even further, all the way down to $r \rightarrow 0$ in the limit $\alpha \rightarrow 0$. This fact by itself is quite striking.

The simple E-model attractors have a potential of the form $V_{0}\left(1-e^{-\sqrt{\frac{2}{3 \alpha}} \varphi}\right)^{2 n}$. For $n=1, \alpha=1$ it gives the potential of the Starobinsky model, with the prediction $r \approx 0.003$. We will generalize both T-models as well as E-models, which both fit the data from Planck very well, to describe SUSY breaking and dark energy, at the minimum of the generalized potential.

Other models which make related predictions include the fibre inflation model [35] and the Starobinsky-like models developed in [36]. Note that in figures 1 and 2, as well as in the figures 3 and 5 , we show the predictions for the number of e-foldings $N=60$. But this number may be significantly lower, depending on the mechanism of reheating [37]. This may somewhat change the predictions for $n_{s}$ and $r$. In particular, for $N=50$ the value of $n_{s}$ at $\alpha \lesssim 10$ in all of our models decreases by $\Delta n_{s} \sim 0.0067$.

\section{$2.3 \quad$ Stabilizers}

In supergravity models of inflation, the task of SUSY breaking after inflation is often delegated to the so-called hidden SUSY breaking sector, requiring the addition of new superfields constrained to not participate in inflation. The scalars from such superfields have 


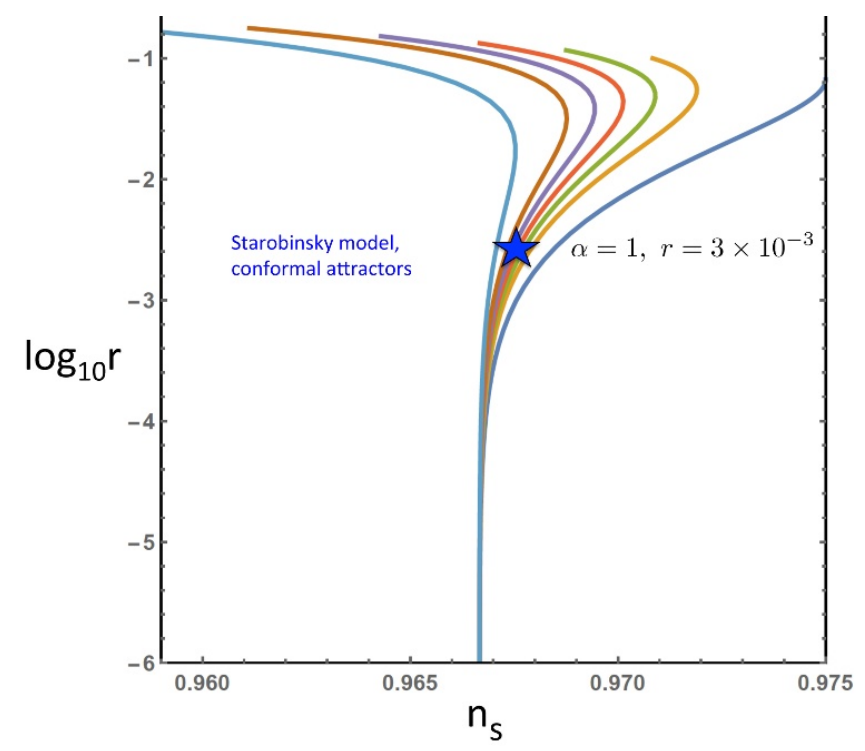

Figure 2. The cosmological observables $\left(n_{s}, r\right)$, in a logarithmic scale in $r$, for simple examples of E-models, with $V=\left(1-e^{-\sqrt{\frac{2}{3 \alpha}} \varphi}\right)^{2 n}$ with $n=(1 / 2,3 / 4,7 / 8,1,3 / 2,2,3)$ starting from the right, increasing to the left, with the vertical line for $n=1$ in the middle. When $\alpha \rightarrow \infty$ the models approach the ones with $\varphi^{2 n}$ potentials. The attractor line, common for all $n$, starts below $r \approx 10^{-3}$ and goes down, unlimited.

to be strongly stabilized, so as to not affect the inflation driven by the inflaton sector of the model. In this paper we describe models of chaotic inflation with the inflaton chiral superfield, and with a nilpotent superfield stabilizer. ${ }^{1}$ This new approach to generic SUSY breaking was suggested recently in [5] using generic supergravity models including the inflaton multiplet as well as a nilpotent multiplet $[45,46]$. Note that the non-inflaton goldstino multiplet plays an important role for consistency of inflation, including stabilization of the second scalar belonging to the inflaton multiplet. This was explained in [49, 50] developing on the pioneering work [51]. In these models the glodstino multiplet was a 'stabilizer' superfield and was a standard chiral superfield.

\subsection{Shift symmetry and $\mathrm{Z}, \mathrm{T}$, and $\Phi$ variables}

The inflationary models made with a shift-symmetric canonical Kähler potential, and controllable supersymmetry breaking have been studied in [5-8]. The basic feature of all such models is as follows. At the potential's minimum supersymmetry is spontaneously broken. With the simplest choice of the Kähler potential, the models are given by $K=$ $\frac{1}{2}(\Phi-\bar{\Phi})^{2}+S \bar{S}, W=g(\Phi)+S f(\Phi), S^{2}(x, \theta)=0$, where the superpotential depends on two functions of the inflaton field $\Phi$. The difference with earlier models [49-51], is the presence of an $S$-independent function $g(\Phi)$ in $W$ and the requirement that $S$ is nilpotent. The

\footnotetext{
${ }^{1}$ The nilpotent multiplet describes the Volkov-Akulov fermionic goldstino multiplet with non-linearly realized spontaneously broken supersymmetry [38, 39]. The relation to chiral nilpotent multiplets was studied in [40-43]. In cosmology we use the recent implementation of nilpotent multiplets suggested in [44]. These nilpotent multiplets are deeply related to the physics of the D-branes [45-48].
} 
mass of the gravitino at the minimum of the potential, $W=m_{3 / 2}=g(0)$, is non-vanishing in these new models, and SUSY is broken in the goldstino direction with $D_{S} W=M \neq$ 0 . In [49-51] the mass of the gravitino was vanishing. Typically the minimum of the potential is these models had an unbroken supersymmetry in Minkowski minima. But in new models in [5-8] with $g(\Phi) \neq 0$ we find instead either de Sitter or Minkowski minima with spontaneously broken SUSY.

From the point of view of string theory and $\mathcal{N} \geq 2$ spontaneously broken supergravity, another class of Kähler potentials, such as $K=-3 \alpha \ln (T+\bar{T})$, is more interesting due to their geometric nature and symmetries. The same models in Poincaré disk variables are given by $K=-3 \alpha \ln (1-Z \bar{Z})$. It is particularly important that these models have a boundary of the moduli space at

$$
Z \bar{Z} \rightarrow 1, \quad Z \rightarrow \pm 1, \quad T \rightarrow 0, \quad T^{-1} \rightarrow 0
$$

where $T=\frac{1+Z}{1-Z}, \quad T^{-1}=\frac{1-Z}{1+Z}[10,13,25]$. Inflation takes place near the boundary which leads to an attractor behavior when many models lead to the same inflationary predictions. A simple way to explain it is to refer to a geometric nature of the kinetic terms of the form

$$
\left.3 \alpha \frac{\partial T \partial \bar{T}}{(T+\bar{T})^{2}}\right|_{T=\bar{T}=t}=\frac{3 \alpha}{4}\left(\frac{\partial t}{t}\right)^{2}=\frac{3 \alpha}{4}\left(\frac{\partial\left(t^{-1}\right)}{t^{-1}}\right)^{2}
$$

The kinetic term has a pole behavior near $t^{-1} \rightarrow 0$, near the boundary of the moduli space $T^{-1} \rightarrow 0$. This explains why the potentials can be changed without a change in cosmological observables and $r$ depends on the residue of the pole, i.e. on $\alpha$ [23]. We may therefore change our potentials by small terms depending on $t^{-1}$ without changing the observables during inflation.

We study these models here. They can use either the Poincaré disk variables $Z \bar{Z}<1$ or the half-plane variables $T+\bar{T}>0$. We will also use the set of variables discussed in [27], where

$$
T=e^{\sqrt{\frac{2}{3 \alpha}} \Phi}, \quad Z=\tanh \frac{\Phi}{\sqrt{6 \alpha}} .
$$

In the context of our moduli space geometry the variables $\Phi$ represent the Killing adapted frame where the metric is inflaton independent. We will therefore call them Killing variables.

Our purpose here is to generalize the models in [10-13] to break $\mathcal{N}=1$ SUSY spontaneously. The new models with $S^{2}(x, \theta)=0$, which are compatible with established cosmological data and designed to be compatible with the future data on $r$ and $m_{3 / 2}$ will depend on four parameters: $\alpha$, describing the Kähler geometry, $M$, defining the scale of SUSY breaking by goldstino $D_{S} W=M$, and $\mu$, related to scale of inflationary energy and $b$. The role of $b$ is the following: at the minimum

$$
V=\left(b^{2}-3\right) \frac{M^{2}}{b^{2}}, \quad \Rightarrow \quad b^{2}=3, \quad V=0 .
$$

It shows that in $\mathcal{N}=1 \mathrm{~d}=4$ supergravity with a nilpotent goldstino multiplet generic de Sitter minima require a universal condition that the goldstino energy $M^{2}$ exceeds the 
negative gravitino contribution to energy where $m_{3 / 2}^{2}=\frac{M^{2}}{b^{2}}$.

$$
V=M^{2}-3 m_{3 / 2}^{2}>0 \text {. }
$$

We keep here generic values of the parameter $b^{2}>3$ which allow generic de Sitter vacua of the string landscape type, including the case

$$
\Lambda=M^{2}-3 m_{3 / 2}^{2}=\left(1-\frac{3}{b^{2}}\right) M^{2} \sim 10^{-120} .
$$

\section{$3 \quad$ Killing-adapted $\alpha$-attractor supergravity models}

We study here the following $\mathcal{N}=1$ supergravity models, which can be described in disk geometry coordinates of the moduli space $Z$,

$$
K=-3 \alpha \log (1-Z \bar{Z})+S \bar{S}, \quad S^{2}(x, \theta)=0, \quad W=\tilde{A}(Z)+S \tilde{B}(Z) .
$$

The geometry has the $\mathrm{SU}(1,1)$ symmetry

$$
d s^{2}=K_{Z \bar{Z}} d Z d \bar{Z}=-3 \alpha \frac{d Z d \bar{Z}}{(1-Z \bar{Z})^{2}} .
$$

Alternatively, we can use the half-plane coordinates $T$

$$
K=-3 \alpha \log (T+\bar{T})+S \bar{S}, \quad S^{2}(x, \theta)=0, \quad W=\tilde{G}(T)+S \tilde{F}(T) .
$$

The geometry has an $\mathrm{SL}(2, \mathbb{R})$ symmetry

$$
d s^{2}=K_{T \bar{T}} d T d \bar{T}=-3 \alpha \frac{d T d \bar{T}}{(T+\bar{T})^{2}} .
$$

In both cases, at $S=0$ the geometry is associated with the Poincare disk or half plane geometry where $3 \alpha=R_{E}^{2}$ corresponds to the radius square of the Escher disk [25].

We will now perform a Kähler transformation $[26,27]$ so that our new Kähler potential is inflaton shift-symmetric. First we use the original disk and half-plane variables and redefine the Kähler and superpotentials as follows

$$
K=-\frac{3}{2} \alpha \log \left[\frac{(1-Z \bar{Z})^{2}}{\left(1-Z^{2}\right)\left(1-\bar{Z}^{2}\right)}\right]+S \bar{S}, \quad S^{2}(x, \theta)=0, \quad W=A(Z)+S B(Z) .
$$

where

$$
A(Z)+S B(Z)=\left(1-Z^{2}\right)^{-3 \alpha / 2}(\tilde{A}(Z)+S \tilde{B}(Z)) .
$$

In half-plane case

$$
K=-\frac{3}{2} \alpha \log \left[\frac{(T+\bar{T})^{2}}{4 T \bar{T}}\right]+S \bar{S}, \quad S^{2}(x, \theta)=0, \quad W=G(T)+S F(T) .
$$

where

$$
G(T)+S F(T)=T^{-3 \alpha / 2}(\tilde{G}(T)+S \tilde{F}(T))
$$


Since we have performed a Kähler transform of the type

$$
\begin{array}{lll}
K \rightarrow K+\frac{3 \alpha}{2} \log \left[\left(1-Z^{2}\right)\left(1-\bar{Z}^{2}\right)\right], & W \rightarrow\left(1-Z^{2}\right)^{-3 \alpha / 2} W & \bar{W} \rightarrow\left(1-\bar{Z}^{2}\right)^{-3 \alpha / 2} \bar{W} \\
K \rightarrow K+\frac{3 \alpha}{2} \log [4 T \bar{T}], & W \rightarrow T^{-3 \alpha / 2} W & \bar{W} \rightarrow \bar{T}^{-3 \alpha / 2} \bar{W} .
\end{array}
$$

the geometry did not change, it is still given by (3.2) and (3.4), respectively.

Our next step is to switch to moduli space coordinates (2.4) where the metric is manifestly inflaton-independent. The choice of coordinates $Z=\tanh \frac{\Phi}{6 \alpha}$ and $T=e^{\sqrt{\frac{2}{3 \alpha}} \Phi}$ in the disk/half-plane geometry corresponds to a Killing-adapted choice of coordinates where the metric does not depend on $\varphi=\operatorname{Re} \Phi$. We find that in these coordinates with Killing variables $\Phi=\varphi+i \vartheta$

$$
K=-3 \alpha \log \left[\cosh \frac{\Phi-\bar{\Phi}}{\sqrt{6 \alpha}}\right]+S \bar{S}
$$

and

$$
d s^{2}=-3 \alpha \frac{d Z d \bar{Z}}{(1-Z \bar{Z})^{2}}=-3 \alpha \frac{d T d \bar{T}}{(T+\bar{T})^{2}}=\frac{\partial \Phi \partial \bar{\Phi}}{2 \cos ^{2}\left(\sqrt{\frac{2}{3 \alpha}} \operatorname{Im} \Phi\right)} .
$$

The superpotential is now

$$
W=A\left(\tanh \frac{\Phi}{\sqrt{6 \alpha}}\right)+S B\left(\tanh \frac{\Phi}{\sqrt{6 \alpha}}\right)=G\left(e^{\sqrt{\frac{2}{3 \alpha}} \Phi}\right)+S F\left(e^{\sqrt{\frac{2}{3 \alpha}} \Phi}\right) .
$$

Note that in our models $\vartheta=0$ during inflation and therefore the new holomorphic variable $\Phi$ during inflation becomes a real canonical variable $\varphi$. This is also easy to see from the kinetic terms in these variables, which are conformal to flat,

$$
d s^{2}=\frac{d \varphi^{2}+d \vartheta^{2}}{2 \cos ^{2} \sqrt{\frac{2}{3 \alpha}} \vartheta} .
$$

At $\vartheta=0$ they are both canonical $\left.d s^{2}\right|_{\vartheta=0}=\frac{d \varphi^{2}+d \vartheta^{2}}{2}$. Thus, we will work with $\alpha$-attractor models (3.1), (3.3) in the form

$$
K=-3 \alpha \log \left[\cosh \frac{\Phi-\bar{\Phi}}{\sqrt{6 \alpha}}\right]+S \bar{S}, \quad W=G\left(e^{\sqrt{\frac{2}{3 \alpha}} \Phi}\right)+S F\left(e^{\sqrt{\frac{2}{3 \alpha}} \Phi}\right) .
$$

Here one should keep in mind that our original half-plane variable $T$ is related to $\Phi$ as follows, $T=e^{\sqrt{\frac{2}{3 \alpha}} \Phi}$. We will use the following notation

$$
G\left(e^{\sqrt{\frac{2}{3 \alpha}} \Phi}\right) \equiv g(\Phi), \quad F\left(e^{\sqrt{\frac{2}{3 \alpha}} \Phi}\right) \equiv f(\Phi) .
$$

To summarize, in Killing variables the $\alpha$-attractor supergravity models are

$$
K=-3 \alpha \log \left[\cosh \frac{\Phi-\bar{\Phi}}{\sqrt{6 \alpha}}\right]+S \bar{S}, \quad W=g(\Phi)+S f(\Phi) .
$$

We find that the potential at $\Phi=\bar{\Phi}$ and at $S=0$ is given by

$$
V_{\text {total }}=2\left|g^{\prime}(\varphi)\right|^{2}-3|g(\varphi)|^{2}+|f(\varphi)|^{2},
$$


since the Kähler covariant derivatives are the same as simple derivatives

$$
D_{\Phi} W=\partial_{\Phi} W=g^{\prime}(\Phi), \quad D_{S} W=\partial_{S} W=f(\Phi),
$$

and at $\Phi=\bar{\Phi}, S=0, K=0$ and the inverse kinetic terms $K^{S \bar{S}}=1$ and $K^{\Phi \bar{\Phi}}=2$.

\section{Reconstruction models of inflation with SUSY breaking and de Sitter exit}

In the form (3.17) our $\alpha$-attractor models can be used to provide a de Sitter exit from inflation as well as supersymmetry breaking at the minimum of the potential, without changing any of the advantages in describing inflation. One of the simplest possibilities for such models is to require that

$$
\begin{aligned}
g(\Phi) & =\frac{1}{b} f(\Phi), \\
K & =-3 \alpha \log \left[\cosh \frac{\Phi-\bar{\Phi}}{\sqrt{6 \alpha}}\right]+S \bar{S}, \quad W=\left(S+\frac{1}{b}\right) f(\Phi) .
\end{aligned}
$$

In Killing variables we find that at $\Phi=\bar{\Phi}$ and at $S=0$

$$
D_{\Phi} W=\partial_{\Phi} W=\frac{1}{b} f^{\prime}(\Phi), \quad D_{S} W=\partial_{S} W=f(\Phi) .
$$

The expression for the potential at $\Phi-\bar{\Phi}=S=0$ is now very simple and is given by

$$
V=\left(1-\frac{3}{b^{2}}\right)|f(\varphi)|^{2}+\frac{2}{b^{2}}\left|f^{\prime}(\varphi)\right|^{2} .
$$

Assume that at the minimum of the potential at $\Phi=0$

$$
f(0)=D_{S} W=M \neq 0, \quad f^{\prime}(0)=b D_{\Phi} W=0 .
$$

This means that at the minimum supersymmetry is broken only in the direction of the nilpotent superfield $S$ and unbroken in the inflaton direction, since $b \neq 0$.

We take $b^{2}>3$. This provides an opportunity to have de Sitter vacua with positive cosmological constant $\Lambda$ in our inflationary models so that

$$
\left.V\right|_{\Phi=0}=\Lambda, \quad \Lambda \equiv\left(1-\frac{3}{b^{2}}\right) M^{2}, \quad b^{2}=\frac{3}{1-\frac{\Lambda}{M^{2}}} .
$$

The cosmological constant is extremely small, $\Lambda \sim 10^{-120}$, so we would like to make a choice of $f$ in (4.1) such that the inflationary potential is presented by the second term in (4.4). In such case, with account of $\vartheta=0$ condition we can use the reconstruction method analogous to the one in [6], where it was applied to canonical shift symmetric Kähler potentials with Minkowski vacua. We will show here how to generalize it for de Sitter exit from inflation and our logarithmic Kähler potentials.

If the potential during inflation is expected to be given by the function

$$
V(\varphi)=\mathcal{F}^{2}(\varphi)
$$


we have to take

$$
\partial_{\varphi} f(\varphi)=\frac{b}{\sqrt{2}} \mathcal{F}(\varphi)
$$

and

$$
f(\varphi)=\left.\frac{b}{\sqrt{2}} \int \mathcal{F}(\varphi) \quad f(\varphi)\right|_{\varphi=0}=M .
$$

In these models the value of the superpotential at the minimum defines the mass of gravitino as follows

$$
W_{\min }=\left.\frac{f}{b}\right|_{\Phi=0}=\frac{M}{b}=\frac{M}{\sqrt{3}}\left(1-\frac{\Lambda}{M^{2}}\right)^{1 / 2}=m_{3 / 2},
$$

where $\Lambda=M^{2}-3 m_{3 / 2}^{2}$. The total potential at $\vartheta=0$ is therefore given by

$$
V^{\text {total }}=\Lambda \frac{|f(\varphi)|^{2}}{M^{2}}+|\mathcal{F}(\varphi)|^{2}
$$

with

$$
V_{\min }^{\text {total }}=\Lambda=M^{2}-3 m_{3 / 2}^{2} .
$$

To get from the supergravity model (4.2) to the Planck, LHC, dark energy potential (4.11) requires stabilization of the field $\vartheta$ at $\vartheta=0$. We have checked that for all values of $\alpha$ during inflation, up to slow roll parameters, the main contribution to the mass to Hubble ratio is of the form

$$
\frac{m_{\vartheta}^{2}}{H^{2}} \approx 6 \frac{|f|^{2}}{\left|f^{\prime}\right|^{2}} \gg 1
$$

Here the mass of $\vartheta$ is defined with a proper account taken of the non-trivial kinetic term. Equation (4.13) implies that $\vartheta$ quickly reaches its minimum at $\vartheta=0$ at the bottom of the de Sitter valley, and inflation proceeds due to a slow evolution of $\varphi$. However, near the minimum of the potential, where the slow roll parameters are not small, a more careful evaluation of the mass of $\vartheta$ has to be performed. We will do it in examples below.

\subsection{The simplest T-model with broken SUSY and dS exit}

We would like to have the inflationary part of the the potential to be

$$
V_{\text {infl }}(\varphi)=\alpha \mu^{2} \tanh ^{2} \frac{\varphi}{\sqrt{6 \alpha}}
$$

This means that

$$
\mathcal{F}=\sqrt{\alpha} \mu \tanh \frac{\varphi}{\sqrt{6 \alpha}}
$$

and

$$
f(\varphi)=\sqrt{3} \alpha \mu b \log \left[\cosh \frac{\varphi}{\sqrt{6 \alpha}}\right]+M .
$$

At $\varphi=0$ one has $f(\varphi)=M$. A complete supergravity version of the model is

$$
K=-3 \alpha \log \left[\cosh \frac{\Phi-\bar{\Phi}}{\sqrt{6 \alpha}}\right]+S \bar{S}, \quad W=\left(S+\frac{1}{b}\right)\left[\sqrt{3} \alpha \mu b \log \left[\cosh \frac{\Phi}{\sqrt{6 \alpha}}\right]+M\right] .
$$




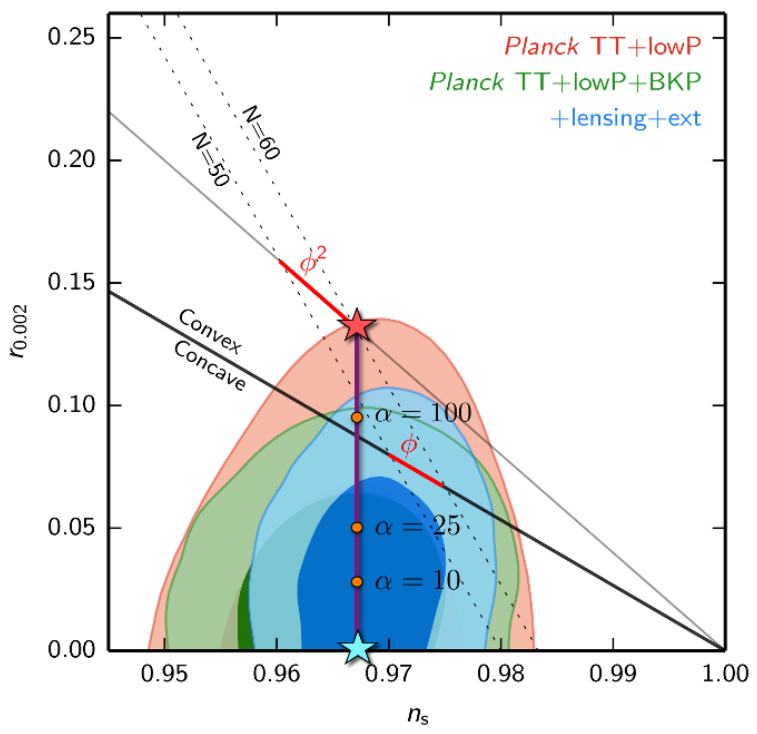

Figure 3. Cosmological predictions of the simplest T-model (4.18) with SUSY breaking and a non-vanishing cosmological constant $\Lambda \sim 10^{-120}$.

The total potential has a part proportional to the cosmological constant $\Lambda$ as well as the second part describing inflation:

$$
V_{\text {total }}=\Lambda \frac{|f(\varphi)|^{2}}{M^{2}}+\alpha \mu^{2} \tanh ^{2} \frac{\varphi}{\sqrt{6 \alpha}} .
$$

The issue of the $\vartheta$ field stabilization which is required to get from (4.17) to (4.18) presents an example of the general case. We find that during inflation $\frac{m_{\vartheta}^{2}}{H^{2}}$ is positive and large, $\vartheta$ quickly reaches 0 . However, near the minimum of the potential, the evaluation of the mass of $\vartheta$ shows that it is positive under condition that $\alpha \gtrsim 0.2$. Thus for $r \gtrsim 10^{-3}$ the model is safe without any stabilization terms even at the de Sitter minimum. For smaller $\alpha$ the bisectional curvature term has to be added to the Kähler potential, to stabilize $\vartheta$. It is given by an expression in disk variables of the form $A(Z, \bar{Z}) S \bar{S}(Z-\bar{Z})^{2}$.

The cosmological predictions of this model are represented by the straight vertical line in figure 1. A more direct comparison with the Planck results is provided by a figure presented in [24], which we reproduce here as figure 3.

Note that this model in disk variables and in a different Kähler frame was already presented in eqs. (3.20) and (3.21) in [24]. An interesting property of the model (4.18) is that the amplitude of scalar perturbations does not depend on $\alpha$ and is determined only by $\mu \approx 10^{-5}$.

\subsection{The simplest E-model with broken SUSY and dS exit}

We are looking at the inflationary $\alpha$ model with

$$
V_{\text {infl }}=m^{2}\left(1-e^{-\sqrt{\frac{2}{3 \alpha}} \varphi}\right)^{2} .
$$


This means that

$$
\mathcal{F}=m\left(1-e^{-\sqrt{\frac{2}{3 \alpha}} \varphi}\right)
$$

and

$$
f(\varphi)=\frac{m b}{\sqrt{2}}\left(\varphi+\sqrt{\frac{3 \alpha}{2}} e^{-\sqrt{\frac{2}{3 \alpha}} \varphi}-1\right)+M .
$$

At $\varphi=0$ one has $f(\varphi)=M$.

Thus our complete model is

$$
K=-3 \alpha \log \left[\cosh \frac{\Phi-\bar{\Phi}}{\sqrt{6 \alpha}}\right]+S \bar{S}, \quad W=\left(S+\frac{1}{b}\right)\left[\frac{m b}{\sqrt{2}}\left(\Phi+\sqrt{\frac{3 \alpha}{2}} e^{-\sqrt{\frac{2}{3 \alpha}} \Phi}-1\right)+M\right] .
$$

The total potential has a part proportional to the cosmological constant $\Lambda$ as well as the second part describing inflation:

$$
V_{\text {total }}=\Lambda \frac{\mid f(\varphi))\left.\right|^{2}}{M^{2}}+m^{2}\left(1-e^{-\sqrt{\frac{2}{3 \alpha}} \varphi}\right)^{2}
$$

The issue of the $\vartheta$ field stabilization which is required to get from (4.22) to (4.23) has been studied separately and again confirms the general case as discussed below eq. (4.12) concerning inflationary part. And again near the minimum of the potential, the evaluation of the mass of $\vartheta$ shows that it is positive under condition that $\alpha>0.2$. For smaller values of $\alpha$, the bisectional curvature term has to be added to the Kähler potential, to stabilize $\vartheta$. It is of the form $A(Z, \bar{Z}) S \bar{S}(Z-\bar{Z})^{2}$ in disk variables. This model for $\alpha=1$ in half-plane variables in case of $\Lambda=0$ was proposed in [9] in eqs. (28), (37). For the generic case of $\alpha \neq 1$ a related model was given in eqs. (4.23), (4.24) in [24].

More general models can be constructed following the rules for this class of models proposed above in eqs. (4.7) - (4.11).

\section{General models of inflation with SUSY breaking and dark energy}

We have learned above how to build supergravity models by reconstructing superpotentials to produce a given choice of the bosonic inflationary potential $V(\varphi)=\mathcal{F}^{2}(\varphi)$ with our logarithmic Kähler potential $K=-3 \alpha \log \left[\cosh \frac{\Phi-\bar{\Phi}}{\sqrt{6 \alpha}}\right]+S \bar{S}$ in Killing variables. The exact answer for $W=g(\Phi)+S f(\Phi)$ can be obtained under condition $g(\Phi)=\frac{1}{b} f(\Phi)$ and requires simply an integration so that $f(\varphi)$ is reconstructed by integration $f(\varphi)=\frac{b}{\sqrt{2}} \int \mathcal{F}(\varphi)$. Obviously this can be carried out in any variables as long as one takes care of the Kähler measure relating the variables used to the functional form of the canonical variables, but it is particularly transparent in Killing-adapted variables as the measure is unity.

Instead of the reconstructing strategy we may start with our models in (3.17) with superpotentials of the form

$$
W=g(\Phi)+S f(\Phi)
$$

without a constraint that $g(\Phi)=\frac{1}{b} f(\Phi)$. In such case the potentials are given by $V_{\text {total }}=$ $2\left|g^{\prime}(\varphi)\right|^{2}-3|g(\varphi)|^{2}+|f(\varphi)|^{2}$. 
Near the minimum of the potential one has to check that we still satisfy the requirements that $D_{S} W=M \neq 0$ and $D_{\Phi} W=0$ to preserve the nice de Sitter exit properties with SUSY breaking as described in eq. (2.5). In these models we end up with more complicated bosonic potentials describing some combination of our $\alpha$-attractor models. However, these models are still capable to fit the cosmological observables as well as providing the level of SUSY breaking in dS vacua with a controllable gravitino mass. Some examples of these models were given in [24], in eqs. (2.4), (3.15) and (2.7), (3.17). Here we will present an example where in disk variables the superpotential is relatively simple whereas the potential is not simple but satisfactory for our purpose. We take the inflaton shift-symmetric Kähler potential and the superpotential of the form

$$
\begin{aligned}
K & =-\frac{3}{2} \alpha \log \left[\frac{(1-Z \bar{Z})^{2}}{\left(1-Z^{2}\right)\left(1-\bar{Z}^{2}\right)}\right]+S \bar{S}, \quad S^{2}(x, \theta)=0, \\
W & =\left(S+\frac{1-Z^{2}}{b}\right)\left(\sqrt{3} \alpha m^{2} Z^{2}+M\right) .
\end{aligned}
$$

The same model in Killing variables $\Phi$, where $Z=\tanh \frac{\Phi}{\sqrt{6 \alpha}}$, is

$$
\begin{aligned}
K & =-3 \alpha \log \left[\cosh \frac{\Phi-\bar{\Phi}}{\sqrt{6 \alpha}}\right]+S \bar{S} \\
W & =\left(\frac{1}{b} \cosh ^{-2}\left(\frac{\Phi}{\sqrt{6 \alpha}}\right)+S\right)\left(\sqrt{3 \alpha} m^{2} \tanh ^{2}\left(\frac{\Phi}{\sqrt{6 \alpha}}\right)+M\right) .
\end{aligned}
$$

The potential at $S=0$ and $\vartheta=0$ has the form $V_{\text {total }}=2\left|g^{\prime}(\varphi)\right|^{2}-3|g(\varphi)|^{2}+|f(\varphi)|^{2}$, where in our case

$$
\begin{aligned}
& g(\varphi)=\frac{1}{b} \cosh ^{-2}\left(\frac{\Phi}{\sqrt{6 \alpha}}\right)\left(\sqrt{3 \alpha} m^{2} \tanh ^{2}\left(\frac{\Phi}{\sqrt{6 \alpha}}\right)+M\right), \\
& f(\varphi)=\sqrt{3 \alpha} m^{2} \tanh ^{2}\left(\frac{\Phi}{\sqrt{6 \alpha}}\right)+M .
\end{aligned}
$$

We have checked that the mass of the field $\vartheta$ is positive everywhere for all $\alpha>0.02$ and that during inflation the ratio $\frac{m_{\vartheta}^{2}}{H^{2}}=6$. This can be also seen from the figure 4 , where we plotted our potential. The inflationary de Sitter valleys are of the same width everywhere for larger and larger values of $\varphi$.

The predictions of this class of models for $n_{s}$ and $r$ practically coincide with the predictions of the models discussed in sections 4.1 and 4.2 for $\alpha=O(1)$. However, at $\alpha \gg 1$ the predictions are somewhat different. We show these predictions in figure 5 by a thin green line for $20>\alpha>1 / 3$ and for the number of e-foldings $N=60$. The top of the line indicated by the dark red star corresponds to $\alpha=20$. The line ends at the pink star corresponding to $\alpha=1 / 3$. We see that the predictions of this model in the large interval $20>\alpha>1 / 3$ belong to the dark blue region favored by the Planck data.

Thus in the last two sections we have presented several supergravity models where $\frac{\delta \rho}{\rho}$, $n_{s}$ and $\Lambda$ take their known observable values, whereas the gravitino mass $m_{3 / 2}$ and the tensor-to-scalar ratio $r$ are free parameters which can take a broad range of values. 


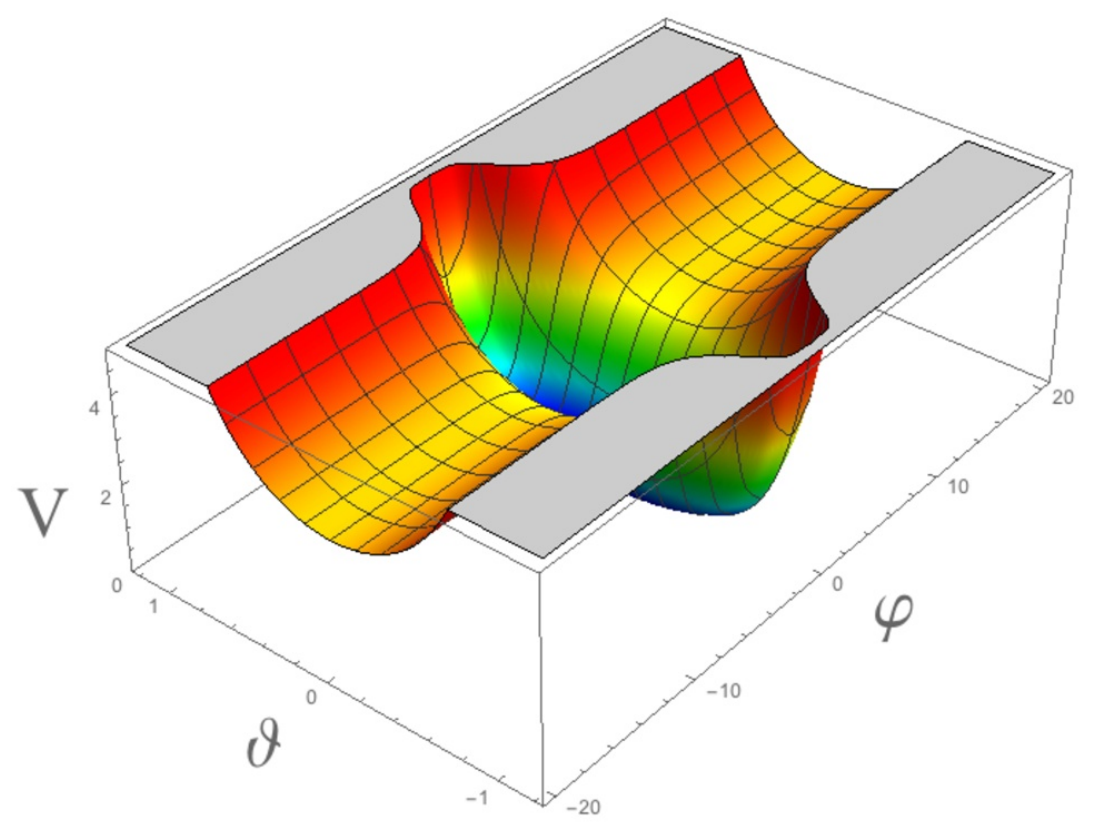

Figure 4. The potential for the supergravity model in eq. (5.3) as a function of $\varphi$ and $\vartheta$. It has a de Sitter minimum at $\varphi=\vartheta=0$ where $V_{\min }=\Lambda$. Supersymmetry is broken at this minimum with $D_{S} W=M$, the mass of gravitino is $m_{3 / 2}^{2}=\frac{M^{2}}{3}\left(1-\frac{\Lambda}{M^{2}}\right)$. The inflationary de Sitter valleys have a nice feature known for models with Minkowski minimum with unbroken SUSY, studied in [27]. These valleys provide nice initial conditions for the inflation to start in these models.

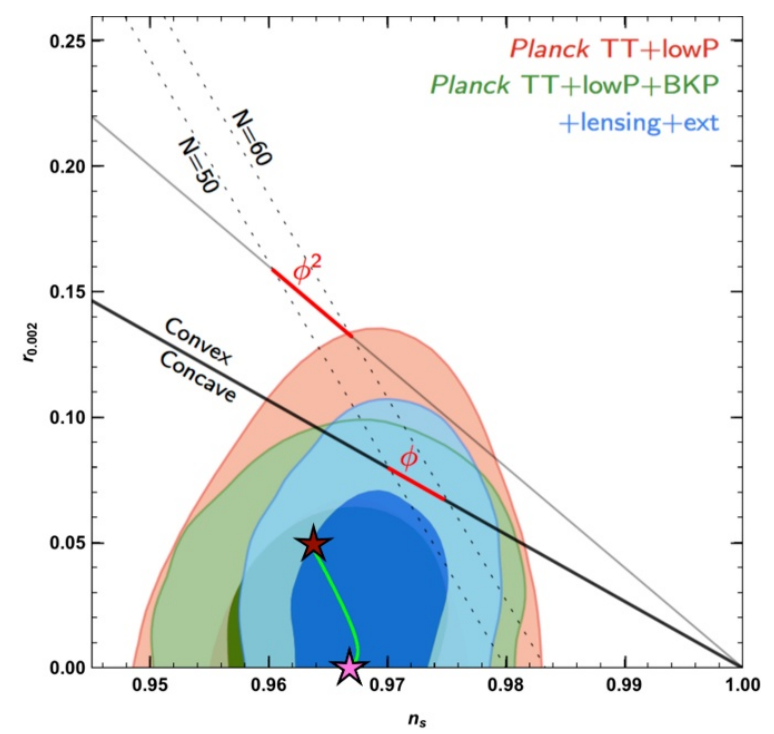

Figure 5. Predictions of the model 5.2 for $20>\alpha>1 / 3$ are shown by the thin green line. The top of the line indicated by the dark red star corresponds to $\alpha=20$. The line ends at the pink star corresponding to $\alpha=1 / 3$. 


\section{Discussion}

In this paper we have pursued a program of describing the main features of the universe evolution, early universe inflation and current acceleration compatible with the data, as well as providing an explanation of the possible origin of the supersymmetry breaking and the mass of gravitino, compatible with the future data from particle physics. Certain features of our four-parameter 'primordial' supergravity models are motivated by the non-perturbative string theory. The origin of the nilpotent superfield $S^{2}(x, \theta)=0$ in these constructions is related to the D-brane physics, where one finds the fermionic Volkov-Akulov goldstino multiplet [38-44] on the world-volume of the D-branes [47, 48].

Our new cosmological models in string theory inspired supergravity suggest a possible bottom-up $\mathcal{N}=1$ supergravity models of inflation which might lead to a successful phenomenology of the early universe and the one which is accelerating now. These models also address the supersymmetry breaking issues. They differ from more traditional string cosmology models which were developed during the last decade, the latest models being discussed in [52-54] and other papers. Our models have fundamental connections to string theory via the nilpotent superfield associated with the fermions on the D-branes. Another connection is via logarithmic Kähler potentials which are required for $\mathcal{N} \geq 2$ supergravity and are present in string theory motivated supergravity. And finally, the value of the positive cosmological constant in our models can be only explained with the reference to the string landscape.

Indeed, the cosmological constant is incredibly small, $\Lambda \sim 10^{-120}$, quantum corrections can significantly change it. However, if string theory allows exponentially many metastable vacua with different values of $\Lambda$, then some of them are bound to belong to the anthropically allowed range not much different from $\Lambda \sim 10^{-120}$ [55-67]. The full string theory description of this scenario, including quantum corrections, is beyond current reach, but one may expect that the resulting theory in the low energy limit should be described by $N=1$ supergravity with some phenomenological Kähler potential $K$ and superpotential $W$. Our goal in this paper was to develop a proper supergravity framework and find potentials $K$ and $W$ which can play this role and simultaneously describe inflation, SUSY breaking and the cosmological constant.

The mass of gravitino, $m_{3 / 2}$, and the level of gravity waves, $r$, are free parameters in our new cosmological models, to be determined by the future experiments. The progress in this direction was based on a better understanding of moduli stabilization and on the use of supergravity models with the universal spontaneous supersymmetry breaking via a fermionic goldstino multiplet. The reason for such universality is the following: the nilpotency condition $S^{2}(x, \theta)=0$ for $S=s+\sqrt{2} \theta \psi_{s}+\theta^{2} F_{s}$ can be satisfied only if $F_{s} \neq 0$. In such case the sgoldstino is not a fundamental scalar anymore but is given by a bilinear combination of fermionic goldstino's divided by the value of the auxiliary field $F_{s}$

$$
s=\frac{\psi_{s} \psi_{s}}{2 F_{s}} .
$$

There is no non-trivial solution if SUSY is unbroken and $F_{s}=0$, i.e. only $s=\psi_{S}=0$ solve the equation $S^{2}(x, \theta)=0$. Thus by requiring to have a fermion Volkov-Akulov 
goldstino nilpotent multiplet in supergravity theory we end up with the universal value of the supergravity potential at its minimum, with $e^{K}\left|F_{S}\right|^{2}=M^{2}$

$$
V=e^{K}\left(\left|F_{S}\right|^{2}-3|W|^{2}\right)=M^{2}-3 m_{3 / 2}^{2}=\Lambda>0 .
$$

The new always positive goldstino contribution originates in an updated version of the KKLT uplifting via the the $\bar{D} 3$ brane, with manifest spontaneously broken supersymmetry $[47,48]$.

Our minimal supergravity models depend on two superfields, one of them typically represented using either a Poincare-disk variable $Z$, or a half-plane variable $T$. A new variable $\Phi$ which we used extensively in this paper describes the same geometry but in a Killing adapted frame where the metric does not depend on the inflaton direction. We call $\Phi$ a Killing variable. We explained the relation between these three holomorphic variables in section 3 . The canonically normalized inflaton in our models is $\varphi=\operatorname{Re} \Phi$. The inflaton partner scalar $\vartheta=\operatorname{Im} \Phi$ is supposed to vanish, which happens automatically during inflation in the models considered in this paper. In all our models in $\Phi$-variables the inflaton shift-symmetric Kähler potential is $K=-3 \alpha \ln \left[\cosh \frac{\Phi-\bar{\Phi}}{\sqrt{6 \alpha}}\right]+S \bar{S}$ and the superpotential is $W=g(\Phi)+S f(\Phi)$. The nilpotent multiplet $S$ does not have fundamental scalars, it has only a fermionic goldstino.

In models with a canonical Kähler potential for the nilpotent multiplet $K=S \bar{S}$ stabilization of $\vartheta$ in all models presented in this paper does not require any additional stabilization terms, as long as $\alpha>0.2$. For smaller $\alpha$ one can stabilize $\vartheta$ by adding a bisectional curvature term to the Kähler potential of the form (in disk variables) $A(Z, \bar{Z}) S \bar{S}(Z-\bar{Z})^{2}$. Thus, in presence of the nilpotent superfield $S$ the problem of stabilization of the direction orthogonal to the inflaton is solved during inflation as well as at the minimum of the potential.

An unexpected benefit from the new tools for moduli stabilization during inflation was realized very recently. Many examples of previously known supergravity models, compatible with current and future cosmological observations, can now easily describe dark energy via tiny de Sitter vacua, and spontaneous breaking of supersymmetry. In this paper we provide examples of such generalizations of $\alpha$-attractor models [10-26]. These models interpolate between various polynomial models $\varphi^{2 m}$ at very large $\alpha$ and attractor line for $\alpha \leq 1$, see figures 1, 2. Therefore they are flexible with regard to data on B-modes, $r$. They provide a seamless natural fit to Planck data. For these kinds of cosmological models we have shown that it is possible to break supersymmetry without an additional hidden sector, with a controllable parameter of supersymmetry breaking. With inflationary scale $\sim 10^{-5} M_{p}$ the scale of supersymmetry breaking can be $M \sim\left(10^{-13}-10^{-14}\right) M_{p}$, compatible with the discovery of supersymmetry at LHC. With $M \gg 100-1000 \mathrm{TeV}$ we will have equally good inflationary models, compatible with an absence of observed supersymmetry at LHC. In fact, such inflationary models are even easier to construct.

In this paper we developed two methods of constructing inflationary models with supersymmetry breaking and de Sitter minimum. One is the reconstruction method in section 4 , which allows to take any desirable inflationary models, in particular our $\alpha$-attractor models, and enhance them by SUSY breaking and a small cosmological constant. An advantage 
of this method, following $[6-9,24]$, is that it is powerful and easy. It requires only a simple integration of a given function. Thus one can obtain nearly arbitrary inflationary potentials, just as it was done in $[49,50]$, so one can fit any set of observational data in the context of supergravity-based models of inflation. Moreover, in all of these models one can introduce SUSY breaking of any magnitude without introducing extra scalars such as Polonyi field. It can be done while preserving all desirable inflationary predictions. Thus from the purely phenomenological point of view, the reconstruction method is a great tool offering us enormous flexibility.

On the other hand, this method does not use specific advantages of the cosmological attractors, including their geometric origin and stability of their predictions with respect to the change of the inflationary potential. In this sense, the method used for deriving the model described in section 5, as well as of some other similar models found earlier in [24], preserves the attractor features of the theory by construction, for all values of the SUSY breaking parameters and arbitrary cosmological constant. Some of the features of these models (the existence of a dS valley of a constant width and depth shown in figure 3) play an important role in solving the initial conditions problem for inflation in these models. The details of this analysis can be found in [27] for $\alpha$-attractor models with a Minkowski minimum and unbroken SUSY. Here we see that in generic models with de Sitter exit and controllable SUSY breaking, initial condition problem for inflation is solved just as in the simpler case studied in [27].

\section{Acknowledgments}

We are grateful to S. Dimopoulos, M. Dine, S. Kachru, J. March-Russell, D. Roest, M. Scalisi, E. Silverstein, F. Quevedo and F. Zwirner for a discussion of cosmology and particle physics related issues. This work was supported by the SITP and by the NSF Grant PHY1316699. RK is also supported by the Templeton foundation grant 'Quantum Gravity Frontiers,' and AL is also supported by the Templeton foundation grant 'Inflation, the Multiverse, and Holography.' JJMC received support from the Templeton foundation grant 'Quantum Gravity Frontiers,' and is supported by the European Research Council under ERC-STG-639729, 'Predictive Quantum Field Theory'.

Open Access. This article is distributed under the terms of the Creative Commons Attribution License (CC-BY 4.0), which permits any use, distribution and reproduction in any medium, provided the original author(s) and source are credited.

\section{References}

[1] A.D. Linde, Chaotic Inflation, Phys. Lett. B 129 (1983) 177 [InSPIRE].

[2] A.D. Linde, Particle physics and inflationary cosmology, Contemp. Concepts Phys. 5 (1990) 1 [hep-th/0503203] [INSPIRE].

[3] Planck collaboration, P.A.R. Ade et al., Planck 2015 results. XIII. Cosmological parameters, arXiv:1502.01589 [INSPIRE]. 
[4] Planck collaboration, P.A.R. Ade et al., Planck 2015 results. XX. Constraints on inflation, arXiv: 1502.02114 [INSPIRE].

[5] R. Kallosh and A. Linde, Inflation and Uplifting with Nilpotent Superfields, JCAP 01 (2015) 025 [arXiv: 1408.5950] [INSPIRE].

[6] G. Dall'Agata and F. Zwirner, On sgoldstino-less supergravity models of inflation, JHEP 12 (2014) 172 [arXiv:1411.2605] [INSPIRE].

[7] R. Kallosh, A. Linde and M. Scalisi, Inflation, de Sitter Landscape and Super-Higgs effect, JHEP 03 (2015) 111 [arXiv: 1411.5671] [INSPIRE].

[8] M. Scalisi, Cosmological $\alpha$-Attractors and de Sitter Landscape, arXiv:1506.01368 [INSPIRE].

[9] A.B. Lahanas and K. Tamvakis, Inflation in no-scale supergravity, Phys. Rev. D 91 (2015) 085001 [arXiv: 1501.06547] [INSPIRE].

[10] R. Kallosh and A. Linde, Universality Class in Conformal Inflation, JCAP 07 (2013) 002 [arXiv: 1306.5220] [INSPIRE].

[11] S. Ferrara, R. Kallosh, A. Linde and M. Porrati, Minimal Supergravity Models of Inflation, Phys. Rev. D 88 (2013) 085038 [arXiv:1307.7696] [InSPIRE].

[12] R. Kallosh, A. Linde and D. Roest, Superconformal Inflationary $\alpha$-Attractors, JHEP 11 (2013) 198 [arXiv: 1311.0472] [INSPIRE].

[13] S. Cecotti and R. Kallosh, Cosmological Attractor Models and Higher Curvature Supergravity, JHEP 05 (2014) 114 [arXiv: 1403.2932] [INSPIRE].

[14] R. Kallosh, A. Linde and D. Roest, Universal Attractor for Inflation at Strong Coupling, Phys. Rev. Lett. 112 (2014) 011303 [arXiv:1310.3950] [INSPIRE].

[15] G.F. Giudice and H.M. Lee, Starobinsky-like inflation from induced gravity, Phys. Lett. B 733 (2014) 58 [arXiv: 1402.2129] [inSPIRE].

[16] R. Kallosh, More on Universal Superconformal Attractors, Phys. Rev. D 89 (2014) 087703 [arXiv: 1402.3286] [INSPIRE].

[17] C. Pallis, Linking Starobinsky-Type Inflation in no-Scale Supergravity to MSSM, JCAP 04 (2014) 024 [arXiv:1312.3623] [INSPIRE].

[18] C. Pallis, Induced-Gravity Inflation in no-Scale Supergravity and Beyond, JCAP 08 (2014) 057 [arXiv: 1403.5486] [INSPIRE].

[19] C. Pallis, Reconciling Induced-Gravity Inflation in Supergravity With The Planck 20138 BICEP2 Results, JCAP 10 (2014) 058 [arXiv:1407.8522] [INSPIRE].

[20] R. Kallosh, A. Linde and D. Roest, Large field inflation and double $\alpha$-attractors, JHEP 08 (2014) 052 [arXiv: 1405.3646] [INSPIRE].

[21] R. Kallosh, A. Linde and D. Roest, The double attractor behavior of induced inflation, JHEP 09 (2014) 062 [arXiv: 1407.4471] [INSPIRE].

[22] B. Mosk and J.P. van der Schaar, Chaotic inflation limits for non-minimal models with a Starobinsky attractor, JCAP 12 (2014) 022 [arXiv:1407.4686] [INSPIRE].

[23] M. Galante, R. Kallosh, A. Linde and D. Roest, Unity of Cosmological Inflation Attractors, Phys. Rev. Lett. 114 (2015) 141302 [arXiv: 1412.3797] [INSPIRE].

[24] R. Kallosh and A. Linde, Planck, LHC and $\alpha$-attractors, Phys. Rev. D 91 (2015) 083528 [arXiv: 1502.07733] [INSPIRE].

[25] R. Kallosh and A. Linde, Escher in the Sky, arXiv:1503.06785 [InSPIRE]. 
[26] J.J.M. Carrasco, R. Kallosh, A. Linde and D. Roest, Hyperbolic geometry of cosmological attractors, Phys. Rev. D 92 (2015) 041301 [arXiv: 1504.05557] [INSPIRE].

[27] J.J.M. Carrasco, R. Kallosh and A. Linde, Cosmological Attractors and Initial Conditions for Inflation, Phys. Rev. D 92 (2015) 063519 [arXiv:1506.00936] [INSPIRE].

[28] E. Cremmer, J. Scherk and S. Ferrara, SU(4) Invariant Supergravity Theory, Phys. Lett. B 74 (1978) 61 [inSPIRE].

[29] E. Bergshoeff, M. de Roo and B. de Wit, Extended Conformal Supergravity, Nucl. Phys. B 182 (1981) 173 [INSPIRE].

[30] M. de Roo, Matter Coupling in N=4 Supergravity, Nucl. Phys. B 255 (1985) 515 [INSPIRE].

[31] S. Ferrara, R. Kallosh and A. Van Proeyen, Conjecture on hidden superconformal symmetry of $N=4$ Supergravity, Phys. Rev. D 87 (2013) 025004 [arXiv: 1209.0418] [InSPIRE].

[32] A.S. Goncharov and A.D. Linde, Chaotic inflation of the universe in supergravity, Sov. Phys. JETP 59 (1984) 930 [INSPIRE].

[33] A.B. Goncharov and A.D. Linde, Chaotic Inflation in Supergravity, Phys. Lett. B 139 (1984) 27 [INSPIRE].

[34] A. Linde, Does the first chaotic inflation model in supergravity provide the best fit to the Planck data?, JCAP 02 (2015) 030 [arXiv:1412.7111] [INSPIRE].

[35] M. Cicoli, C.P. Burgess and F. Quevedo, Fibre Inflation: Observable Gravity Waves from IIB String Compactifications, JCAP 03 (2009) 013 [arXiv:0808.0691] [INSPIRE].

[36] J. Ellis, D.V. Nanopoulos and K.A. Olive, Starobinsky-like Inflationary Models as Avatars of No-Scale Supergravity, JCAP 10 (2013) 009 [arXiv:1307.3537] [INSPIRE].

[37] J. Ellis, M.A.G. Garcia, D.V. Nanopoulos and K.A. Olive, Calculations of Inflaton Decays and Reheating: with Applications to No-Scale Inflation Models, JCAP 07 (2015) 050 [arXiv: 1505. 06986] [INSPIRE].

[38] D.V. Volkov and V.P. Akulov, Possible universal neutrino interaction, JETP Lett. 16 (1972) 438 [INSPIRE].

[39] D. Volkov and V. Akulov, Is the neutrino a Goldstone particle?, Phys. Lett. B 46 (1973) 109.

[40] M. Roček, Linearizing the Volkov-Akulov Model, Phys. Rev. Lett. 41 (1978) 451 [InSPIRE].

[41] E.A. Ivanov and A.A. Kapustnikov, General Relationship Between Linear and Nonlinear Realizations of Supersymmetry, J. Phys. A 11 (1978) 2375 [InSPIRE].

[42] U. Lindström and M. Roček, Constrained local superfields, Phys. Rev. D 19 (1979) 2300 [INSPIRE].

[43] R. Casalbuoni, S. De Curtis, D. Dominici, F. Feruglio and R. Gatto, Nonlinear Realization of Supersymmetry Algebra From Supersymmetric Constraint, Phys. Lett. B 220 (1989) 569 [INSPIRE].

[44] Z. Komargodski and N. Seiberg, From Linear SUSY to Constrained Superfields, JHEP 09 (2009) 066 [arXiv:0907.2441] [INSPIRE].

[45] S. Ferrara, R. Kallosh and A. Linde, Cosmology with Nilpotent Superfields, JHEP 10 (2014) 143 [arXiv: 1408.4096] [INSPIRE].

[46] I. Antoniadis, E. Dudas, S. Ferrara and A. Sagnotti, The Volkov-Akulov-Starobinsky supergravity, Phys. Lett. B 733 (2014) 32 [arXiv:1403.3269] [INSPIRE].

[47] R. Kallosh and T. Wrase, Emergence of Spontaneously Broken Supersymmetry on an Anti-D3-Brane in KKLT dS Vacua, JHEP 12 (2014) 117 [arXiv:1411.1121] [INSPIRE]. 
[48] E.A. Bergshoeff, K. Dasgupta, R. Kallosh, A. Van Proeyen and T. Wrase, $\overline{\mathrm{D} 3}$ and $d S, J H E P$ 05 (2015) 058 [arXiv: 1502.07627] [INSPIRE].

[49] R. Kallosh and A. Linde, New models of chaotic inflation in supergravity, JCAP 11 (2010) 011 [arXiv: 1008.3375] [INSPIRE].

[50] R. Kallosh, A. Linde and T. Rube, General inflaton potentials in supergravity, Phys. Rev. D 83 (2011) 043507 [arXiv: 1011.5945] [INSPIRE].

[51] M. Kawasaki, M. Yamaguchi and T. Yanagida, Natural chaotic inflation in supergravity, Phys. Rev. Lett. 85 (2000) 3572 [hep-ph/0004243] [INSPIRE].

[52] R. Flauger, L. McAllister, E. Silverstein and A. Westphal, Drifting Oscillations in Axion Monodromy, arXiv: 1412.1814 [INSPIRE].

[53] W. Buchmüller, E. Dudas, L. Heurtier, A. Westphal, C. Wieck and M.W. Winkler, Challenges for Large-Field Inflation and Moduli Stabilization, JHEP 04 (2015) 058 [arXiv: 1501.05812] [INSPIRE].

[54] E. Dudas and C. Wieck, Moduli backreaction and supersymmetry breaking in string-inspired inflation models, JHEP 10 (2015) 062 [arXiv:1506.01253] [INSPIRE].

[55] A.D. Linde, The Inflationary Universe, Rept. Prog. Phys. 47 (1984) 925 [inSPIRE].

[56] A.D. Sakharov, Cosmological Transitions With a Change in Metric Signature, Sov. Phys. JETP 60 (1984) 214 [Zh. Eksp. Teor. Fiz. 87 (1984) 375] [Sov. Phys. Usp. 34 (1991) 409] [INSPIRE].

[57] T. Banks, T C P, Quantum Gravity, the Cosmological Constant and All That..., Nucl. Phys. B 249 (1985) 332 [INSPIRE].

[58] J.D. Barrow and F.J. Tipler. The Anthropic Cosmological Principle, Oxford University Press, New York U.S.A. (1986).

[59] A.D. Linde, Inflation and quantum Cosmology, in: 300 Years of Gravitation, S.W. Hawking and W. Israel eds., Cambridge University Press, Cambridge U.K. (1987).

[60] S. Weinberg, Anthropic Bound on the Cosmological Constant, Phys. Rev. Lett. 59 (1987) 2607 [INSPIRE].

[61] H. Martel, P.R. Shapiro and S. Weinberg, Likely values of the cosmological constant, Astrophys. J. 492 (1998) 29 [astro-ph/9701099] [INSPIRE].

[62] R. Bousso and J. Polchinski, Quantization of four form fluxes and dynamical neutralization of the cosmological constant, JHEP 06 (2000) 006 [hep-th/0004134] [INSPIRE].

[63] E. Silverstein, (A)dS backgrounds from asymmetric orientifolds, hep-th/0106209 [INSPIRE].

[64] A. Maloney, E. Silverstein and A. Strominger, de Sitter space in noncritical string theory, hep-th/0205316 [INSPIRE].

[65] S. Kachru, R. Kallosh, A.D. Linde and S.P. Trivedi, de Sitter vacua in string theory, Phys. Rev. D 68 (2003) 046005 [hep-th/0301240] [INSPIRE].

[66] M.R. Douglas, The Statistics of string/M theory vacua, JHEP 05 (2003) 046 [hep-th/0303194] [INSPIRE].

[67] L. Susskind, The Anthropic landscape of string theory, in Universe or multiverse?, B. Carr eds., Cambridge University Press, Cambridge U.K. (2009), pg. 247 [hep-th/0302219] [INSPIRE]. 